\title{
Sexual rehabilitation with intracavernous alprostadil after radical prostatectomy: Outcomes from a nursing program
}

\author{
Alexandre Gromicho ${ }^{1}$, Pedro Costa ${ }^{2}$, Débora Araújo ${ }^{2}$, Daniela Pereira ${ }^{2}$, Luís Ferraz ${ }^{2}$ \\ ${ }^{1}$ Urology Department, Centro Hospitalar do Funchal, Portugal; \\ ${ }^{2}$ Urology Department, Centro Hospitalar Vila Nova de Gaia/Espinho EPE, Vila Nova de Gaia, Portugal.
}

\begin{abstract}
Summary Introduction and objectives: Erectile dysfunction (ED) is a common complication after radical prostatectomy that affects quality of life. There are several therapeutic options, including intracavernous alprostadil injections (IAI). However, no specific recommendations have been made on the optimal rehabilitation strategy. In this study we evaluated a sexual rehabilitation program (SRP) with IAI for patients with ED after radical prostatectomy, assessing the rate of compliance and reasons for dropout.

Methods: The sexual rehabilitation program (SRP) was offered to all patients who underwent radical prostatectomy from 1 January 2010 to 31 December 2019. The first consultations were performed by a urology specialist nurse, explaining the IAI procedure and possible complications. The program was considered successful when the patients achieved autonomy in the drug preparation with a good injection technique. A medical consultation was performed at 6 months evaluating the IAI usage and adverse events. In case of dropout, a questionnaire about reasons for dropout was performed. The primary endpoint was the rate of compliance and dropout of the program. Secondary endpoints were the reasons for dropout and adverse events.

Results: 340 patients underwent radical prostatectomy at our institution, and 123 patients accepted to participate in the rehabilitation program. A total of 96 patients (78\%) successfully completed the SRP, and at 6 months 60 (62.5\%) still used IAI. Concerning the reasons for dropping out, the most frequent were the need of injectable therapy and pain. Regarding complications, 17 patients $(13.8 \%)$ reported pain related to the injection and 1 patient $(0.8 \%)$ had a priapism, managed with conservative treatment.

Conclusions: Management of post-radical prostatectomy ED by a nursing program achieved good rates of patients' self-injection accomplishment and treatment compliance. Close monitoring for dose adjustment and management of post-injection penile pain is required during the follow-up.
\end{abstract}

KEY WORDS: Radical prostatectomy; Erectile dysfunction; Sexual rehabilitation; Alprostadil.

Submitted 11 August 2021; Accepted 14 October 2021

\section{INTRODUTION}

Radical prostatectomy (RP) is a therapeutic option for patients with localized prostate cancer. Erectile dysfunction (ED) and urinary incontinence are the most common complications, both impairing patients' quality of life (1). ED is classically attributed to the injury of neurovascular bundles. The persistent penile hypoxia due to the loss of physiological erections may lead the cavernous fibrosis and, ultimately, a decline in erectile function (2).

The use of vasoactive drugs may improve tissue oxygenation through increased penile blood flow, and prevent penile fibrosis $(3,4)$. Therefore, early treatment with erectogenic drugs may play an important role in sexual rehabilitation after RP.

Several treatments have been proposed to manage postRP ED: intracavernous alprostadil injections (IAI), phosphodiesterase-5 (PDE-5) inhibitors and vacuum devices $(4,5)$. The IAI has become increasingly common, especially after Montorsi et al. (6) reported that early postoperative administration of alprostadil injections significantly increased the recovery rate of spontaneous erections after $\mathrm{RP}$. The IAI is a complex procedure. It involves the preparation of the syringe, identification of the injection site and correct administration, which requires a minimum of dexterity. Moreover, the need of a penile injection and the loss of spontaneity of the sexual act may lead to patient anxiety. Most studies reported high discontinuation rates of patients treated with IAI, and the lack of explanation and lack of follow-up were important causes of treatment failure and non-compliance (7). So, it is crucial to clearly inform patients and partners about the objectives of the treatment and expectations of sexual recovery. Due to the time required to explain the IAI procedure to the patient during medical consultations and sexual counselling during the follow-up, the management of post-RP ED is challenging. Therefore, some studies reported an erectile rehabilitation program provided by a urology specialist nurse with good results (8).

In this study we evaluated a sexual rehabilitation program (SRP) for patients with ED after RP, assessing the rate of compliance and reasons for dropout.

\section{MATERIALS AND METHODS}

The present study is a single-center, retrospective study. The SRP with IAI was offered to all patients who underwent non-nerve sparing radical prostatectomy from 1 January 2010 to 31 December 2019. A preoperative eval- 
uation included a detailed medical history and sexual habits, assessing the quality of erection, libido, orgasm and ejaculation.

The therapy was offered at the first post-operative consultation and initiated once the patient was interested in sexual rehabilitation, usually after proper continence control. A consultation was performed by a urology specialist nurse and the sexual partner was asked to participate as well. The first consultation evaluated the changes in the sexual habits and expectations with the treatment. The process of preparation and administration IAI, as well as the possible complications associated, were explained. Then the patients were seen once a week and information was collected about the previous administration (efficacy, side effects), the injection technique and the correct dose adjustment if necessary. The quality of erections was evaluated through the Erection Hardness Score (EHS).

The program was considered successful when the patients achieved autonomy in the drug preparation with a good injection technique. In case of dropout, a questionnaire about reasons for dropout was performed. A medical consultation was performed at 6 months after completing the program, evaluating the IAI usage and adverse events. Reasons for abandoning the IAI at 6 months were also reported.

The primary endpoint was the rate of compliance and dropout of the program. Secondary endpoints were the reasons for dropout and adverse events. Data were analysed using SPSS.

\section{RESULTS}

A total of 340 patients underwent radical prostatectomy at our institution, and 123 patients (36.2\%) accepted to participate in the rehabilitation program. Population demographic and clinical features are show in Table 1. The median (IQR) age of the cohort was 63 (60-67) years old. The median time (IQR) between the surgery and the rehabilitation program was 6.8 (3.6-11.2) months. All patients reported being sexually active before the surgery, with 118 (95.6\%) without preoperative erectile dysfunction symptoms. $36.6 \%$ of the patients were initially treated with PDE-5 inhibitors, without success. The first consultations were performed with the patient and sexual partner in 72 cases (58.5\%). The alprostadil dose distribution is represented on Table 2 and 111 patients (90.2\%) achieved erection hard enough for sexual intercourse after IAI (EHS 3 or 4). In general, a minimum of 3 $( \pm 0.7)$ nursing consultations were performed before patients successfully completed the program. A total of 27 patients (22\%) dropped out over the first consultations and did not complete the rehabilitation program. Of the 96 patients who completed the initial rehabilitation program, 60 (62.5\%) still used intracavernous alprostadil at 6 months. The reasons for dropping out are described on Table 3. In most cases was the need of injectable therapy. The second most frequent reason was injection pain, despite adjusting to the lowest effective dose. Regarding complications, 17 patients (13.8\%) reported pain related to the injection and 1 patient $(0.8 \%)$ had a priapism, managed with conservative treatment.

There was no significant difference in drop-out rates with
Table 1.

Population characteristics.

\begin{tabular}{|l|c|}
\hline Characteristics $(\mathbf{n}=\mathbf{1 2 3}$ ) & Statistic \\
\hline Age (years), median (IQR) & $63(60-67)$ \\
\hline Pre-operative erectile dysfunction symptoms, $\mathbf{n}(\%)$ & 4 \\
\hline Diabetes mellitus, $n(\%)$ & 13 \\
\hline Previous radiotherapy, $n(\%)$ & 14.6 \\
\hline Previous PDE-5 inhibitors, $n(\%)$ & 36.6 \\
\hline Time between surgery and rehabilitation program (months), median (IQR) & $6.8(3.6-11.2)$ \\
\hline Urinary incontinence, $n(\%)$ & 60.2 \\
$\cdot$ No & 32.5 \\
$\cdot$ Mild & 6.5 \\
$\cdot$ Moderate to severe & \\
\hline PDE-5: phosphodiesterase-5. & \\
\hline
\end{tabular}

Table 2.

Alprostadil dose distribution.

\begin{tabular}{|l|c|}
\hline Alprostadil lowest effective dose (ug) & $\mathbf{N}(\%)$ \\
\hline 5 & $10(8.4)$ \\
\hline 10 & $60(50.4)$ \\
\hline 15 & $2(1.7)$ \\
\hline 20 & $47(39.5)$ \\
\hline
\end{tabular}

Table 3.

Reason for dropping out the rehabilitation program.

\begin{tabular}{|l|c|}
\hline Injectable therapy & N (\%) \\
\hline Injection pain & $22(37.3)$ \\
\hline Loss of follow up & $12(20.3)$ \\
\hline Lack of sexual interest & $8(13.6)$ \\
\hline Urinary incontinence & $7(11.9)$ \\
\hline Lack of treatment efficacy & $3(5.1)$ \\
\hline
\end{tabular}

age, diabetes mellitus, previous radiotherapy, previous PDE-5 inhibitors, time between surgery-rehabilitation program and urinary incontinence $(p>0.05)$.

\section{Discussion}

Radical prostatectomy is one of the most frequent therapeutic options used for the management of patients diagnosed with localized prostate cancer. However, this treatment has a negative effect on patients' quality of life, particularly affecting sexual life. In fact, some studies concluded that sexual dysfunction was an independent determinant of worse general health-related quality of life after primary treatment for prostate cancer (9). Most of the studies published in the literature evaluates the effectiveness of drugs used in erectile function recovery, but few data are available concerning the protocols and drug compliance. Intracavernous alprostadil remains the main treatment for erectile rehabilitation after radical prostatectomy, improving sexual function also in patients treated with non-nerve sparing technique (10). The beginning of IAI and patient follow-up can become problematic due to the overload of medical consultations. For these reasons 
our department created a program aimed at sexual rehabilitation, provided with the help of urology specialist nurses.

In our study, $78 \%(\mathrm{n}=96)$ of the patients who agreed to participate successfully completed SRP. They were able to successfully self-administer the IAI and achieved the ability to adjust the correct doses. In $58.5 \%$ the nursing consultations were performed with the couple, highlighting the role of spouses/partner in the sexual rehabilitation. The revaluation at 6 months after the program concluded that 60 patients (62.5\%) still used IAI. Regarding the reasons for dropping out, the most frequent were the need of injectable therapy (patients with fear of needles and patients who refuse to undergo injectable therapy) and pain. Interestingly, the cost of the drug was never stated as a reason for dropping out. Regarding patients who abandoned therapy at 6 months, it should be noted that 4 patients $(12.9 \%)$ were able to achieve erection without IAI. Therefore, despite being a potentially effective treatment, the fact that it is an injectable treatment was a major limitation for these patients. Pain was reported in only 17 patients (13.8\%), especially in the first consultations, but was a major reason for dropping out in only 9 patients. The cause of post-IAI pain is not well known and its management is challenging. Patients were recommended to take analgesics 1 hour before the injection and reduce to the lowest effective dose, but in some cases that was not satisfactory. Other strategies reported in the literature were combining the IAI with a numbing product or the use a mixture of vasoactive drugs such as Trimix (combination of alprostadil, phentolamine and papaverine) $(11,12)$.

The combination allows lower doses of each drug, reducing the adverse events. However, none of these combination drugs have a clinical authorization in the treatment of erectile dysfunction and are not available in Portugal.

The dropout rate at the end of the program and at 6 months were $22 \%$ and $37.5 \%$, respectively. For standard rehabilitation with intracavernous alprostadil, drop-out rates of $41-68 \%$ have been reported, most occurring during the first three months (1). So, our results demonstrate lower discontinuation rates than those reported for standard rehabilitation therapy, but comparable with other studies of IAI in which nursing and sexual counselling was performed and maintained $(8,13,14)$. Also post-IAI pain rate was lower than in other studies. Taken together, these results may indicate that a SRP was a key factor for increasing the motivation of the couples and treatment compliance, as well for minimizing the adverse events.

Although not being a primary outcome, the efficacy of IAI was also evaluated. After the first consultation, $90 \%$ of the patients achieved erections hard enough for sexual intercourse.

Our results are comparable with other studies, which reported success rates ranged from $70 \%$ to $95 \%$ (3, 9, 13). Similarly, the percentage of patients without preoperative erectile dysfunction was quite high for a population with a median age of 63 years old. A possible explanation is the fact that younger patients with no preoperative symptoms of erectile dysfunction were the most motivated to initiate sexual rehabilitation. Also, the erectile dysfunction symptoms were not evaluated through validated questionnaires and the final results may not be accurate.

Regarding the time between the surgery and the rehabilitation program, it was approximately 7 months. The reasons for the delay were not reported, but it could be due to the necessity of adjuvant radiotherapy, hormonal therapy or transient urinary incontinence, discouraging some patients from undertaking SRP. On the other hand, patient's anxiety concerning the oncologic outcomes is usual in the first medical consultations, having a negative impact on the motivation to initiate SRP.

Limitations of the study were described previously throughout the discussion, such as the absence of validated questionnaires evaluating the preoperative erectile dysfunction symptoms and injection-related pain, and the delay to initiate the ERP. Another limitation is related to the retrospective single-center, single-arm and nonrandomized design. More robust evidence is needed from multicenter, randomized and controlled trials to establish a standard sexual rehabilitation program to these patients in the future.

\section{Conclusions}

Management of post-radical prostatectomy ED by a nursing program achieved good rates of patients' self-injection accomplishment and treatment compliance, which are the key components of sexual rehabilitation. Close monitoring for dose adjustment and management of postinjection penile pain is required during the follow-up.

\section{REFERENCES}

1. Salonia A, Bettocchi C, Carvalho J, et al. EAU Guidelines on Sexual and Reproductive Health. EAU Guidel. 2021.

2. Mulhall JP, Slovick R, Hotaling J, et al. Erectile dysfunction after radical prostatectomy: Hemodynamic profiles and their correlation with the recovery of erectile function. J Urol. 2002; 167:1371-5.

3. Raina R, Agarwal A, Zippe CD. Management of erectile dysfunction after radical prostatectomy. Urology. 2005; 66:923-9.

4. Montorsi F, Briganti A, Salonia A, et al. Current and future strategies for preventing and managing erectile dysfunction following radical prostatectomy. Eur Urol. 2004; 45:123-33.

5. Kim JH, Lee SW. Current status of penile rehabilitation after radical prostatectomy. Korean J Urol. 2015; 56:99-108.

6. Montorsi F, Guazzoni G, Strambi LF, et al. Recovery of spontaneous erectile function after nerve-sparing radical retropubic prostatectomy with and without early intracavernous injections of alprostadil: results of a prospective, randomized trial. J Urol. 1997; 158:1408-10

7. Titta M, Tavolini IM, Dal Moro F, et al. Sexual counseling improved erectile rehabilitation after non-nerve-sparing radical retropubic prostatectomy or cystectomy - Results of a randomized prospective study. J Sex Med. 2006; 3:267-73.

8. Yiou $R$, Khodari M, Lingombet $O$, et al. Évaluation d'un programme infirmier d'education thérapeutique pour les injections intra-caverneuses d'alprostadil après prostatectomie radicale. Prog Urol. 2011; 21:283-7.

9. Gontero P, Fontana F, Zitella A, et al. A prospective evaluation of efficacy and compliance with a multistep treatment approach for 
erectile dysfunction in patients after non-nerve sparing radical prostatectomy. BJU Int. 2005; 95:359-65.

10. Moussa M, Papatsoris A, Abou Chakra M, et al. Erectile dysfunction post radical cystectomy. The role of early rehabilitation with pharmacotherapy in nerve sparing and non-nerve sparing group: A randomized, clinical trial. Arch Ital Urol Androl. 2021; 93:58-64.

11. Schramek P, Plas EG, Hübner WA, Pflüger H. Intracavernous injection of prostaglandin E1 plus procaine in the treatment of erectile dysfunction. J Urol. 1994; 152:1108-10.
12. Mulhall J, Land S, Parker M,, et al. The use of an erectogenic pharmacotherapy regimen following radical prostatectomy improves recovery of spontaneous erectile function. J Sex Med. 2005; 2:532-40.

13. Polito M, D’anzeo G, Conti A, Muzzonigro G. Erectile rehabilitation with intracavernous alprostadil after radical prostatectomy: Refusal and dropout rates. BJU Int. 2012; 110:1-4.

14. Yiou R, Cunin P, De La Taille A, et al. Sexual rehabilitation and penile pain associated with intracavernous alprostadil after radical prostatectomy. J Sex Med. 2011; 8:575-82.

\section{Correspondence}

Alexandre Gromicho, MD (Corresponding Author)

alexandrepgromicho@gmail.com

Urology Department, Centro Hospitalar do Funchal, Portugal

Adress: Av. Luís de Camões 57, 9000-177, Funchal (Portugal)

Pedro Costa, MD

pedro_r_costa@hotmail.com

Débora Araújo, MD

deboracerqueiraaraujo@gmail.com

Daniela Pereira, MD

filipa.pereira27@gmail.com

Luís Ferraz, MD

ferrasluis@gmail.com

Urology Department, Centro Hospitalar Vila Nova de Gaia/Espinho EPE,

Vila Nova de Gaia (Portugal) 